\title{
Classical analysis of correlated multiple ionization in strong fields
}

\author{
Bruno Eckhardt ${ }^{1}$ and Krzysztof Sacha ${ }^{1,2}$ \\ 1 Fachbereich Physik, Philipps Universität Marburg, D-35032 Marburg, Germany \\ ${ }^{2}$ Instytut Fizyki im. Mariana Smoluchowskiego, Uniwersytet Jagielloński, ul. Reymonta 4, PL-30-059 Kraków, Poland
}

(November 15, 2018)

\begin{abstract}
We discuss the final stages of the simultaneous ionization of two or more electrons due to a strong laser pulse. An analysis of the classical dynamics suggests that the dominant pathway for non-sequential escape has the electrons escaping in a symmetric arrangement. Classical trajectory models within and near to this symmetry subspace support the theoretical considerations and give final momentum distributions in close agreement with experiments.
\end{abstract}

\section{INTRODUCTION}

Classical models for atomic processes can provide useful insights in situations where quantum effects are not prominent, as for instance in the dynamics of highly excited states or in multiphoton absorption processes. Microwave ionization of hydrogen and other atoms with a single valence electron [1] 3 , localized wave packet dynamics or electron scattering off atoms come to mind [4]. They provide a natural starting point for semiclassical investigations that include, at least approximately, quantum interference effects. In particular the ionization of Rydberg atoms in linear and elliptically polarized microwave fields has received considerable attention and similarities and differences between classical and quantal behaviour have been sorted out in great detail [1] 3]. In most cases only a single electron is considered and sufficient to interpret the observations.

Interactions between electrons seem to play an important role in multiphoton multiple ionization in strong laser fields. Experiments show that the yield of multiply charged ions is much higher than can be expected on the basis of an independent electron model [5],6]. More recently, it has been noted that the electrons can escape non-sequentially and that they are correlated in their final state [7 10]. This correlation in the final state came as a big surprise and it is our main objective here to discuss a classical model for it.

\section{THE MODEL}

The full process of multiphoton multiple ionization is quite complicated and involves many steps. A plausible model relevant for the field intensities of the experiments is the rescattering mechanism 11 18]. Before the pulse arrives, the atom is in its ground state. Then one electron escapes from the atom, most likely by tunnelling through the Stark barrier. This electron is then accelerated by the field and can be reflected back towards the atom. During this impact energy is transfered to other electrons, perhaps lifting them above the ionization threshold or bringing them close enough so that tunnelling is again possible. If not enough energy is provided at this stage, perhaps another rescattering process can follow until eventually multiple ionization takes place or the pulse disappears. However, before the escape to multiple ionization all excited electrons pass close to the nucleus where they interact strongly with the each other and with the Coulomb attraction. During this phase their (classical) motion is very fast compared to the field oscillations and an adiabatic analysis, keeping the field fixed, can be applied. Morevoer, because of the strong interaction all memory of the previous motion is lost, so that the initial state for the multiple ionization event is a statistical distribution of electrons close to the nucleus. Our discussion starts once this intermediate cloud of excited electrons has formed. We do not consider the process by which it has been generated; for instance, one might imagine exposing ions to both an electric field and an electron beam.

The arguments that follow focus on two electron escape but can easily be extended to discuss multiple ionization, as indicated below. The Hamiltonian then has as usual the kinetic energy of the electrons, their mutual repulsion, the attraction to the core and the potential due to the oscillating electric field. In many experiments the recoil momentum of the ion is measured, and given the extremely small momenta of the photons it is possible to calculate it as the sum of the momenta of the electrons.

Initially, there is no field and the atom is in its ground state. In the final state, after the pulse is turned off, both electrons are free and have positive total energy. Not all the energy difference has to be provided by the impacting electron since there can be additional acceleration by the field after the electrons escape from the core region. However, within the adiabatic assumption motivated above, the energy content of the intermediate electron cloud has to be high enough to let both electrons escape from the immediate vicinity of the nucleus. Without field this implies positive energy, but if the field is on and non-zero, a Stark saddle forms some distance away from the nucleus and the electrons can escape over it. The rapid acceleration downfield will then pull the electrons out and feed in the energy needed to remain asympotically free once the pulse is gone.

The Stark saddle that forms in the field provides a focus and a bottleneck for the electrons which they have to cross in order to leave the atom. All electrons see the same saddle and would like to cross it, but if they 
try to do simultaneously, as suggested by the observed electron correlations, their mutual repulsion gets in their way. Suppose that one electron is slightly ahead of the other when running up the hill towards the Stark saddle: the one that is ahead has the advantage that the repulsion with the companion pushes it uphill, whereas the one behind not only has to fight the attraction to the nucleus but also the repulsion from the one ahead. Their interaction is perfectly balanced if they cross the saddle side by side, with reflection symmetry with respect to the field axis. The previous considerations suggest that deviations from this symmetric configuration are amplified and cannot lead to simultaneous ionization. The arguments used here are similar to the ones advanced by Wannier in his analysis of double ionization upon electron impact [19,20].

Therefore, we propose that near the threshold for double ionization the dominant path leading to nonsequential double ionization has both electrons escape symmetrically with respect to the field axis. If more than two electrons are ionized simultaneously the natural extension is that they form a regular n-gon in a plane perpendicular to the field axis.

\section{SYMMETRIC DOUBLE IONIZATION}

With the field pointing along the $x$-axis and two electrons confined to the plane $z=0$ their coordinates in the symmetric subspace are $(x, y, 0)$ and $(x,-y, 0)$ in position and $\left(p_{x}, p_{y}, 0\right)$ and $\left(p_{x},-p_{y}, 0\right)$ in momenta. The classical Hamilton function for this geometry then is (in atomic units)

$$
H\left(p_{x}, p_{y}, x, y, t\right)=p_{x}^{2}+p_{y}^{2}+V(x, y, t)
$$

with potential energy

$$
V(x, y, t)=-\frac{4}{\sqrt{x^{2}+y^{2}}}+\frac{1}{2 y}+2 F x f(t) \cos (\omega t+\phi)
$$

and the pulse shape

$$
f(t)=\sin ^{2}\left(\pi t / T_{d}\right)
$$

where the duration of the pulse is taken to be four field cycles, $T_{d}=8 \pi / \omega$. The rescattering of the electrons leads to a highly excited complex of total energy $\tilde{E}$ which every now and then is close to the symmetric configuration described by the Hamiltonian (1). Any configuration on this energy shell (for some fixed time $t$ ) as well as any phase $\phi$ of the field is equally likely, and the experimental observations are averages over initial conditions and phases.

Fig. 11 shows equipotential lines for the potential (2) at a maximum of the field for $F=0.137$ a.u., corresponding to an intensity of $6.6 \cdot 10^{14} \mathrm{~W} / \mathrm{cm}^{2}$. The saddle is located along the line $x=r_{s} \cos \theta$ and $y=r_{s} \sin \theta$ with $\theta=\pi / 6$ or $5 \pi / 6$ and at a distance $r_{s}^{2}=\sqrt{3} /|F f(t) \cos (\omega t+\phi)|$. For the above mentioned field the saddle has an energy of $V_{s}=-1.69$ a.u..

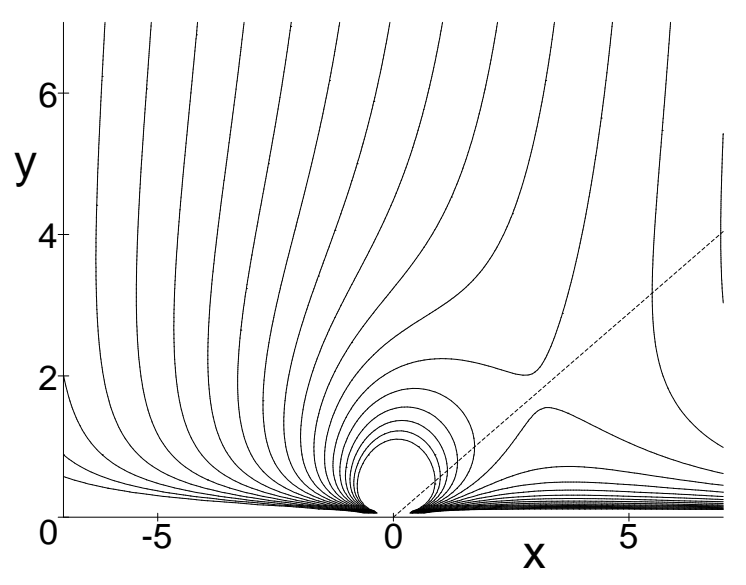

FIG. 1. Adiabatic potential $V(x, y, t)$ for fixed time $t$ in the symmetric subspace. The saddle moves along the dashed line.

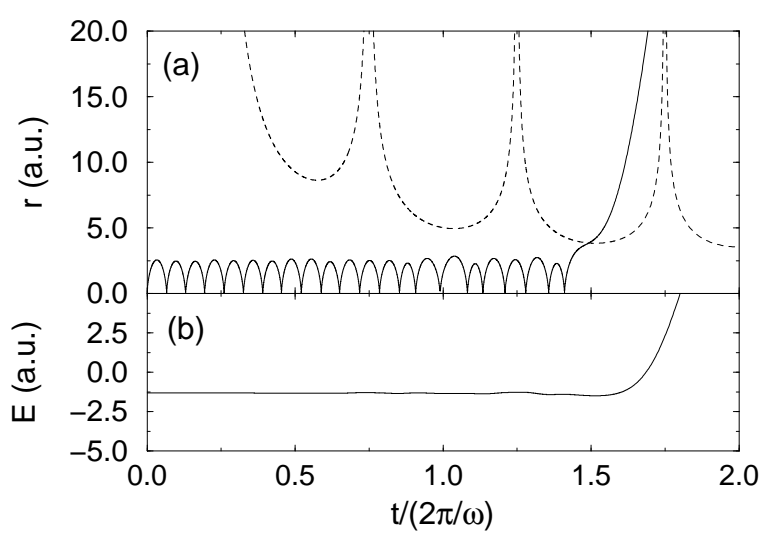

FIG. 2. Trajectories in the symmetric subspace. One frame shows the time evolution of the radial distance $r(t)$ (continuous line) together with the instantaneous position of the barrier (dashed line) and the other shows the energy.

Sample trajectories within the symmetric configuration are shown in Fig. 2. It is evident that they cross the saddle during a maximum of the field and that once on the other side the energy increases rapidly. This acceleration is accompanigned by a rapid separation from the nucleus, so that during field reversals the electrons will not return to the nuclues and will remain essentially free.

A quantity of direct experimental interest is the distribution of momenta of the ion, estimated as $\mathbf{p}_{\text {Ion }} \approx$ $-\left(\mathbf{p}_{1}+\mathbf{p}_{2}\right)$ [7]. The many realization of the exper- 
iment and the unknown details of the initial conditions can be modelled by averaging over all initial conditions of prescribed energy and all phases of the field. The results for peak field strength $F=0.137$ a.u. are shown in Fig. 3 . For initial energy $\widetilde{E}=-0.58$ a.u. the final distribution of momenta clearly shows the double hump structure also seen in experiment. The perpendicular momentum of a single electron show a clear suppression near zero momentum and a long tail. The suppression for small momenta is a clear sign of electron repulsion. We are not aware of expeperimental data on this distribution.
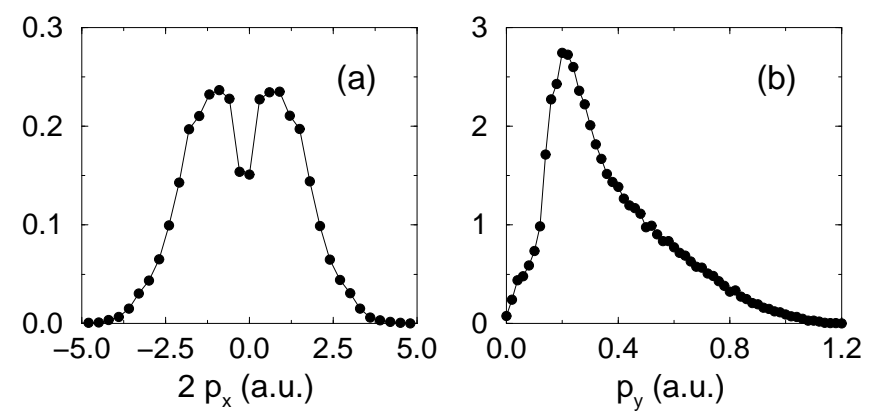

FIG. 3. Final momentum distributions for $F=0.137$ a.u. and initial energy $\tilde{E}=-0.58$ a.u.: (a) ion momentum parallel to the field axis and (b) perpendicular momentum of one electron.

\section{THE SADDLE AND THREE-DIMENSIONAL MOTIONS}

Within the symmetric subspace mentioned before the position of the saddle separating trapped motion from ionized motion is clear. And as in many models of chemical reactions it has one unstable direction that defines the reaction coordinate and a stable motion perpendicular to the reaction coordinate. However, in the space of six degrees of freedom of the full 3-d two electron motion and in the adiabatic approximation for the field the stability analysis of the saddle reveals two unstable directions and four stable ones. The stable directions are of minor importance: if excited they persist in the neighborhood of the saddle as some uncoupled finite amplitude motions. The second unstable direction besides the reaction coordinate is responsible for the importance of the symmetric subspace. Motion leading away from this symmetric subspace will typically have one electron escaping and the other returning to the nucleus. This corresponds to single ionization. The electron returned to the nucleus may have enough energy to ionize in the next step or may gain additional energy from the field to ionize later. Either way, the electrons do not escape symmetrically and simultaneously, so that there are no correlations between the two outgoing electrons and the ionization is sequential.

Without going into the technical details of this analysis, we can illustrate some of these features with trajecto- ries started slightly outside the symmetry plane (Fig. 4).
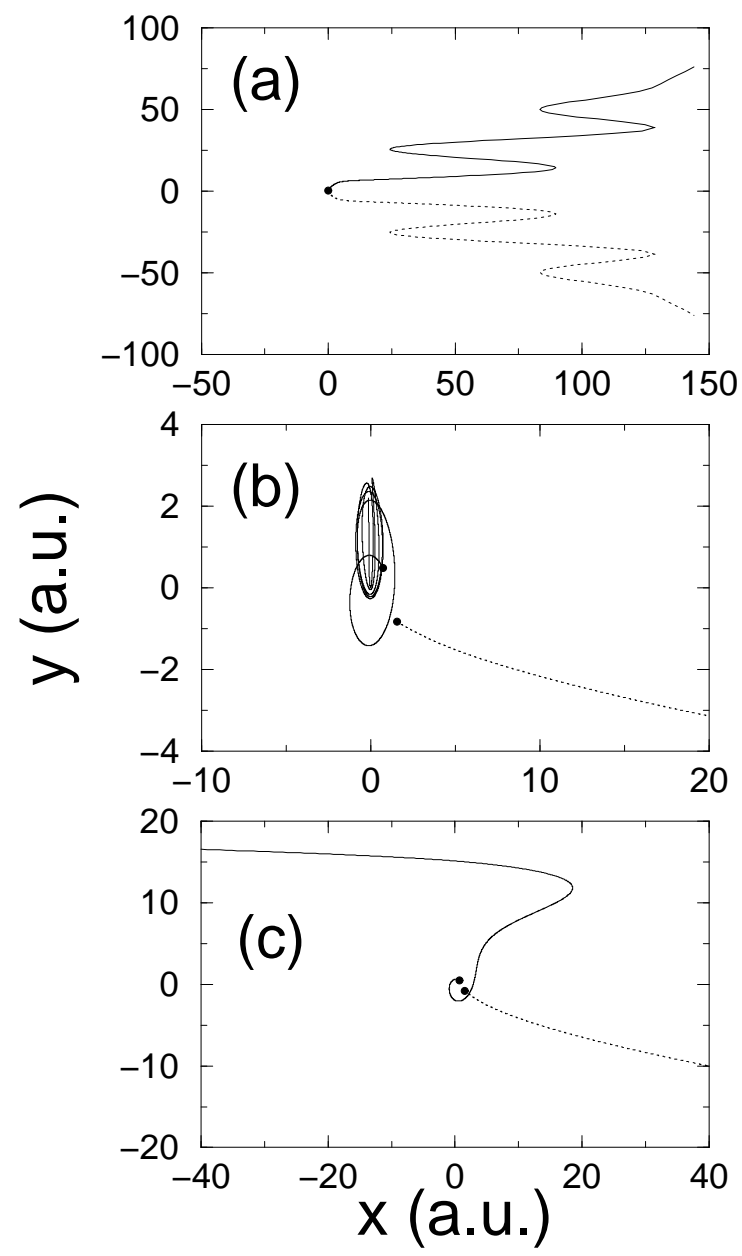

FIG. 4. Trajectories of electrons outside the symmetric subspace for $\tilde{E}=-0.58$ a.u.. Initial positions are close to the saddle and marked by heavy dots; the electrons are distinguished by dotted and continuous tracks. Frame (a) shows for reference the ionization in the symmetric subspace. Frame (b) shows a case where outside this symmetric subspace one electron escapes and the other falls back to the ion. Frame (c) shows an example of sequential ionization of both electrons in opposite directions.

Fig ta shows initial conditions on the saddle and symmetrically escaping electrons. For some deviation from symmetry, one electron escapes, the other remains trapped to the nucleus (Fig. 4b). It is possible, however, that the second electron picks up enough energy to ionize itself (Fig. 4 4 ). In this figure the loss of correlation between the electrons is evidenced by their escape in opposite directions. 


\section{TRIPLE AND HIGHER IONIZATION}

The model is easily extended to the case of simultaneous removal of more than two electrons [9]. The key assumption is that the process is dominated by a symmetric configuration of the electrons with respect to the field polarization axis. Specifically, we assume that all electrons move in a plane perpendicular to the field and that they obey a $C_{N v}$ symmetry, which generalizes the $C_{2 v}$ symmetry of the previous case. The reflection symmetry limits the momenta to be parallel to the symmetry planes and thus confines the motion to a dynamically allowed subspace in the high-dimensional $N$-body phase space.

With the electric field directed along the $z$-axis the positions of the $N$-electrons are $z_{i}=z, \rho_{i}=\rho$ and $\varphi_{i}=2 \pi i / N$, where $\left(\rho_{i}, \varphi_{i}, z_{i}\right)$ are cylindrical coordinates. The momenta of the electrons are all identical, $p_{\rho, i}=p_{\rho}$, $p_{z, i}=p_{z}$ and $p_{\varphi, i}=0$. For this geometry the classical Hamiltonian for $N$ electrons, for zero total angular momentum along the field axis reads

$$
H\left(p_{\rho}, p_{z}, \rho, z, t\right)=N \frac{p_{\rho}^{2}+p_{z}^{2}}{2}+V(\rho, z, t),
$$

with potential energy

$$
V=-\frac{N^{2}}{\sqrt{\rho^{2}+z^{2}}}+\frac{N(N-1)}{4 \rho \sin (\pi / N)}+N z F f(t) \cos (\omega t+\phi) .
$$

The equipotential curves look very much like the ones shown for two particles and the dynamics is similar. One interesting aspect of this many electron configuration is that it is limited to at most 13 electrons: for larger numbers of electrons the repulsion between overweights the attraction to the nucleus and no saddle configuration can be found.

\section{FINAL REMARKS}

The present considerations suggest that correlated, non-seuqential multiple ionization in strong laser pulses proceeds through a saddle configuration with symmetrically moving electrons. The configurations can be seen analogous to the symmetrically escaping electrons in double ionization without field as discussed many years ago by Wannier. As in that case it is possible to derive a threshold law, which, however, is not only different from his but also much more difficult to observe because of the presence of the laser pulse. Further consequences of the model are under investigation.

Financial support by the Alexander von Humboldt Foundation and KBN under project 2P302B00915 are gratefully acknowledged.
[1] P. M. Koch and K. A. H. van Leeuwen, Phys. Rep. 255, 289 (1995).

[2] D. Delande, in: Les Houches Session LII, Chaos and Quantum Physics 1989, editors M. J. Giannoni, A. Voros and J. Zinn-Justin, (North-Holland, Amsterdam), 665 (1991).

[3] D. Delande and J. Zakrzewski, in: Classical, semiclassical and quantum dynamics in atoms $\mathrm{H}$. Friedrich and B. Eckhardt eds., Lecture notes in physics no.485 (Springer, Berlin) 1997.

[4] J.M. Rost, Phys. Rev. Lett. 72, 1998 (1994); Phys. Rep. 297, 271 (1999)

[5] D.N. Fittinghof, P.R. Bolton, B. Chang, and K.C. Kulander, Phys. Rev. Lett. 69, 2642 (1992)

[6] B. Walker, B. Sheehy, L.F. DiMauro, P. Agostini, K.J. Schafer, and K.C. Kulander, Phys. Rev. Lett. 73, 1227 (1994)

[7] Th. Weber, M. Weckenbrock, A. Staudte, L. Spielberger, O. Jagutzki, V. Mergel, F. Afaneh, G. Urbasch, M. Vollmer, H. Giessen and R. Dörner, Phys. Rev. Lett. 84, 443 (2000)

[8] Th. Weber, M. Weckenbrock, A. Staudte, L. Spielberger, O. Jagutzki, V. Mergel, F. Afaneh, G. Urbasch, M. Vollmer, H. Giessen and R. Dörner, J. Phys. B: At. Mol. Opt. Phys. 33, L1 (2000)

[9] R. Moshammer, B. Feuerstein, W. Schmitt, A. Dorn, C.D. Schöter, J. Ullrich, H. Rottke, C. Trump, M. Wittmann, G. Korn, K. Hoffmann and W. Sandner, Phys. Rev. Lett. 84, 447 (2000)

[10] Th. Weber, H. Giessen, M. Weckenbrock, G. Urbasch, A. Staudte, L. Spielberger, O. Jagutzki, V. Mergel, M. Vollmer, and R. Dörner, Nature 405, 658 (2000).

[11] P.B. Corkum, Phys. Rev. Lett. 71, 1994 (1993)

[12] K.C. Kulander, J. Cooper, and K.J. Schafer, Phys. Rev. A 51, 561 (1995)

[13] B. Walker, B. Sheehy, K.C. Kulander, and L.F. DiMauro, Phys. Rev. Lett. 77, 5031 (1996)

[14] A. Becker and F.H.M. Faisal, J. Phys. B 29, L197 (1996)

[15] A. Becker and F.H.M. Faisal, J. Phys. B 32, L335 (1999)

[16] B. Sheehy, R. Lafon, M. Widmer, B. Walker, L.F. DiMauro, P.A. Agostini, and K.C. Kulander, Phys. Rev. A 58, 3942 (1998)

[17] A. Becker and F.H.M. Faisal, Phys. Rev. A 59, R1742 (1999)

[18] A. Becker and F.H.M. Faisal, Phys. Rev. Lett. 84, 3546 (2000).

[19] G.H. Wannier, Phys. Rev. 90, 817 (1953)

[20] A. R. P. Rau , Phys. Rep. 110, 369, (1984) Z. Phys. Chemie B 19, 203 (1932); Trans. Faraday Soc. 3429, (1938) 